\title{
MMP-sensitive PEG hydrogel modified with RGD promotes bFGF, VEGF and EPC-mediated angiogenesis
}

\author{
LIU OUYANG, YANG DAN, ZENGWU SHAO, SHUHUA YANG, CAO YANG, GUOHUI LIU and DEYU DUAN \\ Department of Orthopaedics, Union Hospital, Tongji Medical College, \\ Huazhong University of Science and Technology, Wuhan, Hubei 430022, P.R. China
}

Received October 23, 2017; Accepted March 26, 2019

DOI: $10.3892 /$ etm.2019.7885

\begin{abstract}
Traumatic soft tissue defects such as bedsores, chronic skin ulcers, limb necrosis, osteonecrosis and other ischemic orthopedic diseases are the most clinically intractable and common problems in orthopedics due to unsatisfactory conventional treatments. The present study designed poly(ethylene glycol; PEG) hydrogels with covalently binded arginylglycylaspartic acid (RGD). Endothelial progenitor cells (EPCs) were encapsulated in the modified hydrogel along with vascular endothelial growth factor (VEGF) and basic fibroblast growth factor (bFGF). Results demonstrated that the modified hydrogel displayed good mechanical properties appropriate for a sustained release carrier. RGD modification significantly promoted EPC biocompatibility. VEGF and bFGF encapsulation enhanced the adhesion of EPCs, promoted the production of extracellular matrix and facilitated EPC proliferation. In addition, bFGF and VEGF induced angiogenesis. The combination of growth factors and EPCs in the hydrogel displayed a strong synergy to improve biocompatibility. The present results provided a potential novel treatment approach for soft tissue defects such as bone exposure, chronic skin ulcers, bedsores, limb necrosis, osteonecrosis and other ischemic diseases.
\end{abstract}

\section{Introduction}

Traumatic soft tissue defects such as bedsores, chronic skin ulcers, limb necrosis, osteonecrosis and other ischemic orthopedic diseases are the most clinically intractable and common issues in orthopedics due to unsatisfactory conventional treatments (1). Interest in therapeutic vasculogenesis has arisen as a potential solution to these problems where endothelial

Correspondence to: Dr Deyu Duan, Department of Orthopaedics, Union Hospital, Tongji Medical College, Huazhong University of Science and Technology, 1277 Jiefang Avenue, Wuhan, Hubei 430022, P.R. China

E-mail: deyuduan@yeah.net

Key words: fibroblast growth factor, vascular endothelial growth factor, hydrogel, tissue engineering scaffold, angiogenesis progenitor cells (EPCs) or stem cells are transplanted into the ischemic site to promote angiogenesis $(2,3)$. EPC-mediated angiogenesis has a unique advantage of not being affected by the function of the original blood vessels and native endothelial cells (ECs). EPCs are widely present in adult bone marrow, and have sustained and pluripotent differentiation abilities. EPCs promote the development of blood vessels by differentiating into mature vascular ECs then secreting vascular growth factors $(4,5)$. EPC-mediated angiogenesis improves blood supply to the ischemic site in both animal and clinical trials $(6,7)$; however, injected growth factors are rapidly cleared from the body and thus any transplanted cells undergo rapid cell death in large numbers due to the lack of nutrients. This phenomenon hinders the clinical applications of EPC-mediated therapeutic angiogenesis.

Hydrogels are promising biocompatible and biodegradable biomaterials used as cell transplant vectors that also have roles as drug release systems and tissue engineered cell scaffolds $(8,9)$. Hydrogels are water-soluble polymer networks capable of absorbing a large amount of water to swell and, after swelling, are capable of maintaining their three-dimensional polymer structure due to physical or chemical cross-linking between polymer chains (10). Due to hydrogels displaying good biocompatibility, water permeability and a high degree of swelling this makes them a promising biomaterial for implantation (11). Since 2000, arginylglycylaspartic acid (RGD) peptides have been used to modify hydrogels in order to improve their biocompatibility (8). The RGD tripeptide is the smallest amino acid sequence recognized by adherent proteins. This sequence binds specifically to cell surface integrin receptors, which mediate cell adhesion, migration and growth. Consequently, the immobilization of RGD directly onto a biomaterial promotes the adhesion of receptor-mediated cells to the surface of the material $(8,9,12)$. In 2009, matrix-metalloproteinase (MMP)-sensitive polyethylene glycol (PEG) hydrogels were used to encapsulate human umbilical vein ECs and thymosin $\beta$-4, a human protein that promotes tissue regeneration. It was determined that the modified hydrogel provide an environment that supports human umbilical vein ECs, reduces apoptosis, increases cell survival rate and achieves a controlled release of thymosin $\beta-4$ (12).

The proliferation, differentiation, maturation, and integration of host blood vessels with EPCs require growth factor involvement such as basic fibroblast growth factor (bFGF) 
and vascular endothelial growth factor (VEGF) $(10,11,13,14)$. Vascular growth factors and EPCs work synergistically to create new blood vessels, capillaries, and the formation of capillary networks in the ischemic area. Seliktar et al (15) combined RGD and multiple growth factors into the PEG hydrogel backbone by covalent binding and found that this design not only slowly releases the growth factors but also promotes the adhesion and growth of ECs on the biomaterial surface.

Scaffolds are critical in EPC-mediated therapeutic vasculogenesis therefore the purpose of the present study was to design modified PEG hydrogels with covalently bound RGD to encapsulate EPCs alongside VEGF and bFGF. The role of the growth factors was to maintain EPC health, integrate with the host blood vessels, and also to ensure the sustained long-term release of vascular growth factors into the wound.

\section{Materials and methods}

Animal study. Twenty male Sprague Dawley rats (6-8 weeks) weighing 180-200 g were obtained from the Experimental Animal Center of Huazhong University of Science and Technology. Animals were housed at constant room temperature with a 12-h light-dark cycle, and free access to a standard rodent diet and water. Protocols involving the use of the animals were approved by the Ethics Committee of Tongji Medical College, Huazhong University of Science and Technology (2016 Institutional Animal Care and Use Committee no. S782). Animal experiments were performed in accordance with the Guide for the Care and Use of Laboratory Animals.

Synthesis of four-arm-polyethylene glycol-vinylsulfone (PEG-VS) monomer reaction. The hydrogel synthesis method was adapted from Seliktar et al (15). Four-arm-PEG powder (cat. no. 4ARM-PEG-OH; Shanghai Jinpan Biotech Co., Ltd.) was dissolved in dichloromethane (DCM; analytical reagent, purity $>99.5 \%$ ) to prepare a $0.2 \mathrm{~g} / \mathrm{ml}$ solution. Then PEG-OH, sodium hydride, and diVS were mixed together in a reaction molar ratio of 1:5:50, respectively. The reactor was wrapped with foil to keep it in the dark for the entire time. The reaction was kept in an argon atmosphere and stirred for 3 days at room temperature. Pure acetic acid ( $\mathrm{pH} 7)$ was used to neutralize the solution following completion of the reaction and all residues were filtered using filter paper. Then cold pure acetic acid was then added in excess to produce a precipitate and the supernatant was discarded. The precipitate was washed using DCM, then subsequently resuspended in DCM, and filtered again to produce a precipitate. DCM was used to wash this precipitation twice to remove the excess diVS. Finally, powder was obtained by overnight freeze vacuum drying at $50^{\circ} \mathrm{C}$ in a lyophilizer (model no.FD-1A-50; Beijing Boyikang Laboratory Instruments Co., Ltd.). Nuclear magnetic resonance spectroscopy $\left({ }^{1} \mathrm{H}-\mathrm{NMR}\right.$; AVANCE III HDNanobay $400 \mathrm{MHz}$ compact NMR system; Bruker BioSpin Corporation) was conducted according to the basic NMR operational manual to assess the VS to acquire a peak at 6.1-6.4.

Formation of MMP-sensitive PEG hydrogels modified with $R G D$. Hydrogel formation was prepared as previously described in the literature $(12,16,17)$. In brief, $330 \mathrm{mg}$ of the prepared four-arm-PEG-VS monomer was dissolved in $2.7 \mathrm{ml}$ Tris base, acetic acid and ethylenediaminetetraacetic acid (TAE; $0.3 \mathrm{M} ; \mathrm{pH} 8$ ) solution, with w/v of $10 \%$ at $4^{\circ} \mathrm{C}$, then stored at the same temperature. A total of $4.7 \mathrm{mg}$ RGD (Ac-GCRDGPQGIWGQDRCG-NH $\mathrm{N}_{2}$;Zoonbio Biotechnology, Co., Ltd.) was dissolved in $3 \mathrm{ml}$ TAE buffer $(0.3 \mathrm{M}$; $\mathrm{pH} 8)$ then $4.7 \mathrm{mg}$ matrix metalloproteinase (MMP) reaction substrate peptide (Zoonbio Biotechnology, Co., Ltd.) was dissolved in $3 \mathrm{ml}$ TAE buffer ( $0.3 \mathrm{M}$; pH 8). Following which, $30 \mu \mathrm{l}$ of RGD TAE solution and $30 \mu \mathrm{l}$ of MMP reaction peptide TAE solution were added to $270 \mu 1$ of four-arm-PEG-VS TAE solution then mixed at $37^{\circ} \mathrm{C}$ for $30 \mathrm{~min}$. Finally, the mixed solution was transferred into a clean penicillin bottle and kept at $37^{\circ} \mathrm{C}$ for $30 \mathrm{~min}$ to form a hydrogel.

Hydrogels with 5-carboxyfluorescein (cat. no. F5008; US Everbright, Inc.)-labeled peptide encapsulated with VEGF (cat. no. 400-31) and bFGF (cat. no. 400-29; both 1.2 ng/well; Peprotech) were manufactured in a similar manner as the aforementioned procedures but the RGD peptides were replaced with 5-carboxyfluorescein fluorescent-labeled peptides then hydrogels were soaked in PBS.

Release curve. The hydrogel was soaked in PBS then ELISAs (cat. no. E-EL-R0091 and E-EL-R0020; Elabscience Biotechnology Co., Ltd.) was used to test the content and stability of bFGF and VEGF every $12 \mathrm{~h}$. MMP-2 (100 and 1,000 $\mathrm{ng}$ ) and MMP-9 (100 and 1,000 ng) were added to the samples following $72 \mathrm{~h}$, then the optical density (OD450) value was measured by ELISA. The release curve was plotted after repeating the previous method to detect the slow-release effect of bFGF and VEGF.

Hydrogel stability and biodegradation test. A hydrogel stability test was conducted where hydrogels were transferred into a 12-well plate, and then soaked in $40 \mathrm{ml}$ PBS in the dark at room temperature. PBS was replaced every day. In order to set up the standard solutions of fluorescent RGD peptides, the concentration of RGD peptide were as follows: 2, 1, 0.5, 0.25 and $0.125 \mu \mathrm{g} / \mathrm{ml}$. Fluorescence intensities at OD505 for both standard solutions and the soaking PBS solution were measured with a fluorescence photometer. To test the degradation behavior of the hydrogel, wet weight hydrogel $(20 \mathrm{mg}$; $\mathrm{n}=3$ ) was dried in a lyophilizer until the weight. Then the dried hydrogels were placed in a penicillin bottle, soaked in PBS on a shaking bed at room temperature. The weight of the hydrogels after lyophilization was measured every day and was used for the degradation rate calculation.

Hydrogel enzymatic degradation test. The hydrogels with fluorescently labeled peptides were digested using a bacterial collagenase solution $(0,0.5,1,2$ active bacterial collagenase units per ml PBS; cat. no. JS10538; Shanghai Jinsui Bio-Technology Co., Ltd.). The free fluorescence-labeled peptide concentration in solution was determined by fluorescence spectrophotometer to plot a hydrogel degradation release curve. The difference between tested weight on each day and initial weight on the first day was obtained. The degradation rate was calculated by calculating the ratio of the aforementioned difference and the initial weight. 
EPC adhesion and proliferation. The EPCs isolated and used in the current study were derived from the rat bone marrow where no vascular ECs are present. EPCs were isolated from rat bone marrow cells, according to a standard procedure (18) and then von Willebrand factor (vWF; forward primer, 5'-GCTCCAGCA AGTTGAAGACC-3', reverse primer, 5'-GCAAGTCACTGT GTGGCACT-3'), kinase insert domain receptor (KDR; forward primer, 5'-CTTTTGGTAGCGGGATGAAA-3', reverse primer, 5'-TCCATTGAAATGGGATTGGT-3'), TEK receptor tyrosine kinase (Tek; forward primer, 5'-ACAATCGTGGACGGCTAT TC-3', reverse primer, 5'-AAGGTCTTTAGGGGCTGGAA-3'), CD34 (forward 5'-ACTTCTGTTGCCTCGGAGAA-3'; reverse 5'-ACGGTTGGGTAAGTCTGTGG-3'), CD133 (forward 5'-CCTCTGGTGGGGTATTTCTT-3'; reverse 5'-AGGTGCTGT TCATGTTCTCC-3'), and nitric oxide synthase 3 (eNOS; forward primer, 5'-CACAGGCATCACCAGGAAGAA-3', reverse primer, 5'-ATACCAGTGGGTCCGAGCA-3') gene expression were determined by semi-quantitative PCR to confirm the success of EPC isolation $(3,19)$. $\beta$-actin (F240; forward primer, 5'-CAC GATGGAGGGGCCGGACTCATC-3' and R240; reverse primer, 5'-TAAAGACCTCTATGCCAACACAGT-3') was used as the reference gene. The $25 \mu \mathrm{l}$ PCR reaction system contained $0.5 \mu \mathrm{l} 10 \mu \mathrm{M}$ forward primer, $0.5 \mu \mathrm{l} 10 \mu \mathrm{M}$ reverse primer, $2 \mu \mathrm{l}$ $2.5 \mathrm{mM}$ dNTP (cat. no. D40300A; Takara), $0.25 \mu \mathrm{l}$ Ex Taq (cat. no. DRR100A; Takara), $2.5 \mu \mathrm{l} 10$ fold Ex Taq E buffer, $1 \mu \mathrm{l}$ cDNA and $\mathrm{ddH}_{2} \mathrm{O}$. The PCR conditions were as follows: Denaturation at $95^{\circ} \mathrm{C}$ for $4 \mathrm{~min}$, then 35 cycles of denaturation at $95^{\circ} \mathrm{C}$ for $4 \mathrm{~min}$, annealing at $54^{\circ} \mathrm{C}$ for $30 \mathrm{sec}$ and extension at $72^{\circ} \mathrm{C}$ for $25 \mathrm{sec}$, and a final extension step of $72^{\circ} \mathrm{C}$ for $4 \mathrm{~min}$. The PCR was conducted on an ABI7900 thermocycler (Applied Biosystems; Thermo Fisher Scientific, Inc.) and products were detected on a $1 \%$ agarose gel with $0.5 \mu \mathrm{g} / \mathrm{ml}$ ethidium bromide. Gels were observed with JY02S Gel Imager (Beijing Junyi Electrophoresis Co., Ltd.) and the acquired gel images were processed by Photoshop CS5 (Adobe Systems, Inc.).

To investigate EPC adhesion and proliferation on the hydrogel, EPCs were inoculated on the RGD modified hydrogel surface at a seeding density of $5 \times 10^{4}$ cells $/ \mathrm{cm}^{2}$. EPCs were cultivated in the incubator with $5 \% \mathrm{CO}_{2}$ at $37^{\circ} \mathrm{C}$ in the modified DMEM (Gibco; Thermo Fisher Scientific, Inc.) with $25 \mathrm{U} / \mathrm{ml}$ 10\% FBS heparin, 2 mmol/l glutamine (both Sigma-Aldrich; Merck KGaA), $20 \mu \mathrm{g} / 1 \mathrm{VEGF}, 10 \mu \mathrm{g} / \mathrm{l} \mathrm{bFGF,} 10 \mu \mathrm{g} / 1$ leukemia inhibitory factor and $10 \mu \mathrm{g} / 1$ epidermal growth factor (all Peprotech). The growth of EPCs on the hydrogels were observed and counted using inverted phase contrast microscope. Blank RGD-modified hydrogel was used as a control.

MTT assay for cell metabolic activity. MTT assay was used to assess the biocompatibility of hydrogels. In brief, EPC density was adjusted to a concentration of $1 \times 10^{5}$ cells $/ \mathrm{ml}$ and $100 \mu \mathrm{l}$ cell suspension was added to the surface of RGD-modified hydrogels and non-RGD-modified hydrogels. The plates were incubated under standard conditions $\left(37^{\circ} \mathrm{C} ; 5 \% \mathrm{CO}_{2}\right)$ overnight. Hydrogels where the cells had been inoculated were carefully removed from the wells. Then $10 \mu \mathrm{M}$ MTT solution was added to samples to assess the adherent cells and the plates were incubated at $37^{\circ} \mathrm{C}$ for $4 \mathrm{~h}$. The supernatant was discarded and $100 \mu \mathrm{l}$ of DMSO was added to each well. The plates were placed on a shaking bed for $10 \mathrm{~min}$. The absorbance was read at $570-595 \mathrm{~nm}$ at $0,4,12,24,48$, and $72 \mathrm{~h}$.
EPC culture experiments encapsulated in PEG hydrogel encapsulated with bFGF and VEGF. EPCs were seeded into the hydrogel as previously described (20). The hydrogel was modified according to the previous procedure. The initial EPC seeding density was $5 \times 10^{5} /$ well on a 12 -well plate. bFGF and VEGF were added into each well at a concentration of $1.2 \mathrm{ng} /$ well, respectively. MMP-mediated bFGF and VEGF release rates were detected by bFGF and VEGF ELISA kit (cat. no. E-EL-R0091 and E-EL-R0020, respectively; Elabscience Biotechnology Co., Ltd.) with the method adapted from Kraehenbuehl et al (12). For samples for flow cytometry detection, collected cells were first digested by trypsin-EDTA then washed twice with PBS. After being re-suspended with $500 \mu \mathrm{l}$ binding buffer, $5 \mathrm{ml}$ Annexin V-FITC and $5 \mu \mathrm{l}$ propidinm iodide were added to the cells. The samples were then kept in dark for 5-15 min at room temperature. Viable cells were detected by flow cytometry with by flow cytometry with Annexin V-FITC Apoptosis kit (cat. no. KG108; Nanjing KeyGen Biotech Co., Ltd.). The percentage analysis was finished on Epics XL-4 Flow Cytometer with System II ${ }^{\mathrm{TM}}$ software (version 3.0; both from Beckman Coulter, Inc.).

Reverse transcription-quantitative (RT-q)PCR was performed to determine the expression levels of platelet/endothelial cell adhesion molecule 1 (PECAM1), CD34, KDR, Tek, angiopoietin-2 and protocadherin (pcdh12) genes after 7 days of cultivation. Total RNA from rat EPCs was isolated using TRIzol reagent (cat. no. 15596-018; Invitrogen; Thermo Fisher Scientific, Inc.) and the converted to cDNA with the RevertAid $^{\mathrm{TM}}$ First Strand cDNA Synthesis kit (cat. no. K1622; Fermentas; Thermo Fisher Scientific, Inc.) following manufacturer's protocol. The cDNA was diluted 10-fold in double distilled water and the reaction conditions were: $50^{\circ} \mathrm{C}$ for $2 \mathrm{~min}$, followed by 40 cycles of $95^{\circ} \mathrm{C}$ for $30 \mathrm{sec}$ and $60^{\circ} \mathrm{C}$ for $30 \mathrm{sec}$. qPCRs were conducted on Real PCR machine (ABI7900; Applied Biosystems; Thermo Fisher Scientific, Inc.) using Maxima SYBR-Green/Fluorescein qPCR Master Mix (cat. no. K0241; Fermentas; Thermo Fisher Scientific, Inc.). The reaction system for each gene includes $4 \mu 1$ 10-fold diluted cDNA of the target gene, $10 \mu \mathrm{l}$ Maxima SYBR-Green/Fluorescein qPCR Master Mix (2X), $5.2 \mu \mathrm{l} \mathrm{H}_{2} \mathrm{O}, 0.4 \mu \mathrm{l} 100 \mu \mathrm{M}$ forward primer (PECAM1: 5'-ATCAGCACCACCTCGAAATC-3'; CD34; Tek; KDR; angiopoietin-2: 5'-GGCGAGGAGTCCAACTACAG-3'; and pcdh12: 5'-TCTGGTGCTGACTGCCTATG-3') and $0.4 \mu 1$ $100 \mu \mathrm{M}$ reverse primer (PECAM1: 5'-CTTTTTGTCCACGGT CACCT-3'; CD34; Tek; KDR; angiopoietin-2: 5'-AAGTTG GAAGGACCACATGC-3'; and pcdh12: 5'-CACATGCTTGCC AAAGAAGA-3'). $\beta$-actin 240 was used as the reference gene. The results were quantified using the $2^{-\Delta \Delta \mathrm{Cq}}$ method (21).

The chick chorioallantoic membrane (CAM) method was adapted from Valdes et al (22) to investigate the function of bFGF and VEGF encapsulated within the hydrogel on angiogenesis by scoring the newly formed angiogenic vessels (23). Chicken embryos (Wuhan Institute of Bioloigcal Products, Co., Ltd.) with similar weights were obtained from fresh fertilized eggs $(50 \pm 5 \mathrm{~g})$ after they were opened on a cleaned surface without breaking the embryos. Experimental samples were divided into three groups: Control, modified hydrogel without bFGF or VEGF, and modified hydrogel with both bFGF and VEGF. Each group had three biological replicates. The tested hydrogels were carefully placed in an avascular region between 

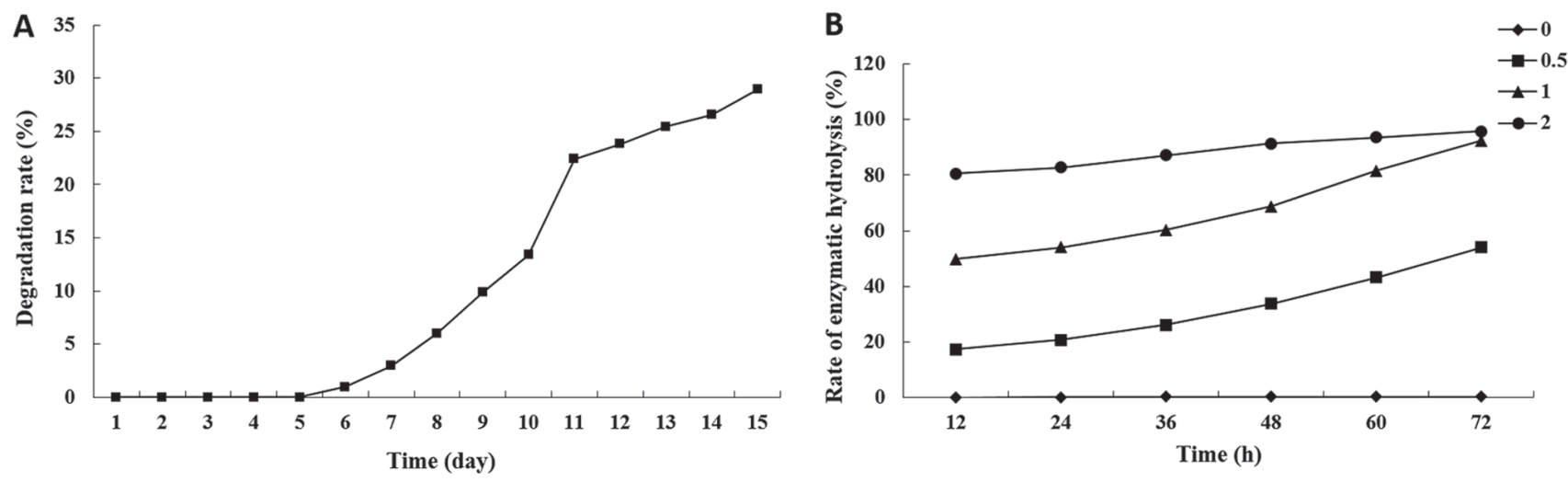

Figure 1. Hydrogel degradation profile. (A) Degradation rate of polyethylene glycol hydrogel over 15 days. (B) Enzymatic hydrolysis rate of different concentrations of collagenase $(0,0.5,1$ and 2 active $\mathrm{U} / \mathrm{ml})$ over $72 \mathrm{~h}$.

Table I. Endothelial progenitor cell number counted in the wells with polyethylene glycol hydrogel.

\begin{tabular}{lcc}
\hline Well number & RGD-modified & Non RGD-modified \\
\hline 1 & 305 & 34 \\
2 & 321 & 42 \\
3 & 318 & 49 \\
Mean & 314.67 & $41.67^{\mathrm{a}}$ \\
\hline
\end{tabular}

${ }^{\mathrm{a}} \mathrm{P}<0.05$ vs. RGD-modified. RGD, arginylglycylaspartic acid.

two main blood vessels in each chicken embryo. The hydrogels were replaced with fresh gels every three days. After 11 days, angiogenic vessels were counted after the removal of the transparent tested hydrogel, then a $2.5 \mathrm{ml}$ methanol and acetone mixture (equal volume) was added to fix the vessels at room temperature for $20 \mathrm{~min}$. The vessel numbers were counted under a dissecting microscope with a 10 -fold magnification (model no. MZFLIII; Leica Microsystems GmbH).

Statistical analysis. All studies had three replicates for every tested group. Statistical analysis was performed using SPSS v20.0 statistical software platform (IBM Corp.). The cell count data was described by the number of cases and examined by Fisher's exact test. Measurement data were expressed as the mean \pm standard deviation, comparisons between three groups were analyzed by ANOVA, followed by the Tukey's Honest Significant Difference post hoc test, and Student's t-test was used to compare the two groups. $\mathrm{P}<0.05$ was considered to indicate a statistically significant difference.

\section{Results}

Hydrogel degradation profile. PEG hydrogels, which present non-adhesive characteristics to proteins and cells, have been widely used to form a thin coating on the injury site of carotid arteries in vivo $(15,24,25)$. The present results demonstrated that the PEG hydrogels were stable for 6 days, then underwent gradual degradation at a relatively constant rate with $\sim 30 \%$ of the gels degraded by 14 days (Fig. 1A). In the enzymatic degradation test, gels were decomposed by bacterial collagenase. The higher the concentration of bacterial collagenase the faster the rate of enzymatic hydrolysis. The hydrogels stored in buffer without collagenase were not decomposed in first $72 \mathrm{~h}$, and the gels kept in the 2 active $\mathrm{U} / \mathrm{ml}$ collagenase were decomposed by $>95 \%$ in the first $72 \mathrm{~h}$ (Fig. 1B).

$R G D$-modified hydrogel significantly increases EPC adhesion and viability. EPCs were inoculated onto the surface of RGD modified hydrogels at $5 \times 10^{4} \mathrm{cell} / \mathrm{cm}^{2}$. The growth of the EPCs and their adhesion to the hydrogel were observed using an inverted phase contrast microscope. The average number of EPCs on the surface of the well in the RGD-modified hydrogel group was significantly higher compared with that of the control group $(\mathrm{P}<0.001$; Table I). In addition, the viability of EPCs on the gels was assessed with the MTT assay. The number of adherent cells was higher in the RGD-modified group compared with the control one $(\mathrm{P}<0.05)$. In summary, the RGD-modified PEG hydrogel significantly promoted the adhesion and growth of the EPCs.

Release of bFGF and VEGF from hydrogels. Growth factor-based cell therapies are considered to be potentially curative treatment options $(16,26)$. The main limitation of growth factor application in biomedical therapies is their high diffusibility and short half-life in vivo (16). The present study prepared enzyme-sensitive PEG hydrogels containing RGD using the Michael-type addition reaction method (12). The growth factors bFGF and VEGF were encapsulated in the hydrogel and the concentrations in the supernatant were detected by ELISA every $12 \mathrm{~h}$ and compared with standard curves. The standard curves of bFGF and VEGF were drawn by establishing a relationship between actual OD values (the difference between measured OD values and blank OD values) and paired standard concentrations (Fig. 2A and B). The release $\%$ of bFGF and VEGF were both maintained at $41 \%$ over $72 \mathrm{~h}$ (Fig. 2C and D).

Following the first $72 \mathrm{~h}$, MMP-2 and MMP-9 were added to the hydrogels. The release $\%$ of bFGF and VEGF both increased steadily. At $148 \mathrm{~h},>90 \%$ of bFGF and VEGF had been released into the supernatant (Fig. 2C and D). In addition, it was determined that the greater the initial MMP-2 and MMP-9 concentration then the higher the growth factor 

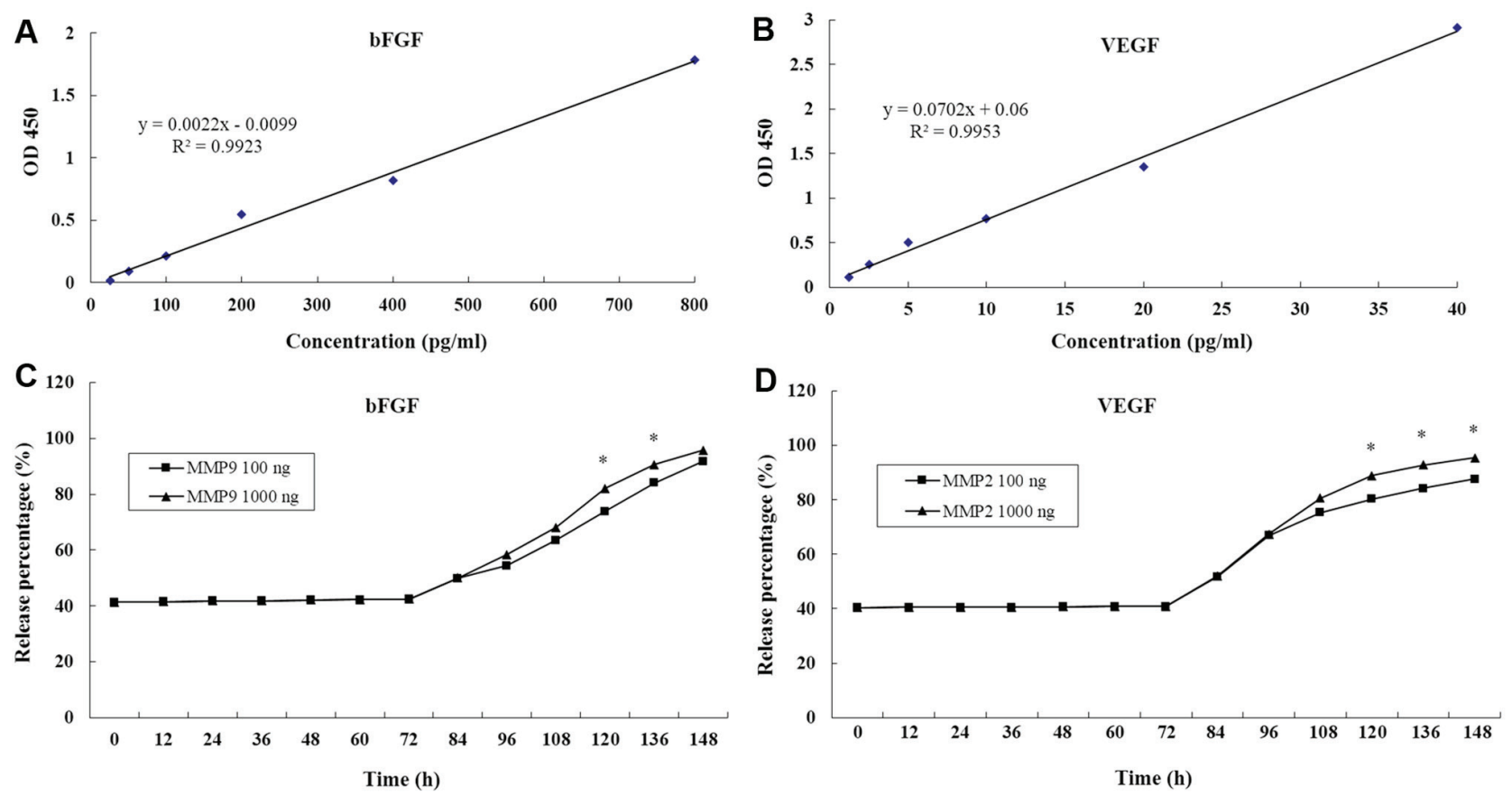

Figure 2. Release of bFGF and VEGF from polyethylene glycol hydrogels up to $148 \mathrm{~h}$. (A) Standard curve of increasing concentrations of bFGF. (B) Standard curve of increasing concentrations of VEGF. (C) bFGF release \% over $148 \mathrm{~h}$ with addition of MMP-9 (100 and 1,000 ng) at $72 \mathrm{~h}$. (D) VEGF release \% over $148 \mathrm{~h}$ with addition of MMP-9 (100 and 1,000 ng) at $72 \mathrm{~h}$. "P<0.05 vs. MMP $100 \mathrm{ng}$ group. bFGF, basic fibroblast growth factor; VEGF, vascular endothelial growth factor; MMP, matrix metalloproteinase; OD, optical density.

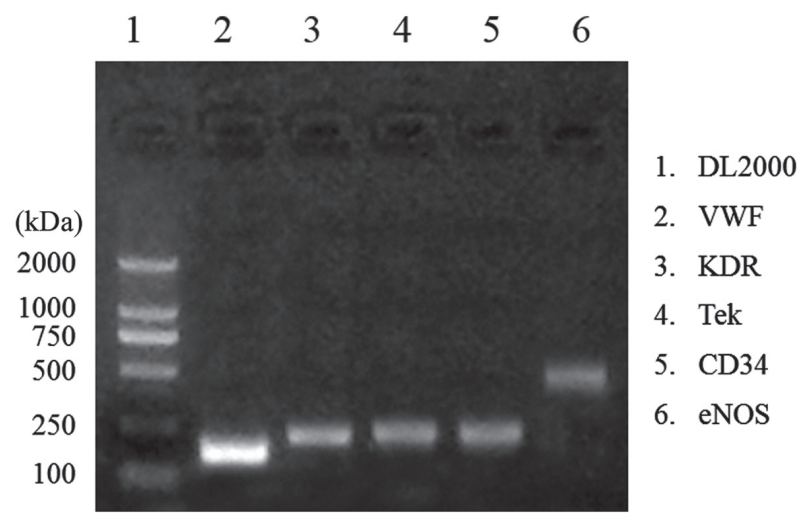

Figure 3. Semi-quantitative PCR results for EPC-related gene expression Gene expression results confirmed the successful isolation of EPCs from rat bone marrow. EPC, endothelial progenitor cells; VWF, von Willebrand factor; KDR, kinase insert domain receptor; Tek, TEK receptor tyrosine kinase; eNOS, nitric oxide synthase 3 .

release \% (Fig. 2). Results suggested that the MMP-sensitive PEG hydrogels maintained a stable release of bFGF and VEGF.

EPC isolation confirmation by semi-quantitative gene expression. The expression of VWF, KDR, Tek, CD34 and eNOS genes are used to determine isolation of EPCs (27). The present gene expression results visualized by $1.5 \%$ agarose gel electrophoresis indicated that EPCs were successfully isolated due to upregulation of the aforementioned genes (Fig. 3).

Encapsulation of bFGF and VEGF within hydrogels significantly increases EPC survival rate. Flow cytometry demonstrated that EPC survival rate in hydrogels encapsulated with bFGF and VEGF was $38.69 \%$ compared with $4.14 \%$ in hydrogels without bFGF and VEGF (P<0.05; Fig. 4). Typically, cells that do not aggregate have a high mortality rate following the first 7 days; however the present results demonstrated that the growth factors promoted EPC cell survival in hydrogels.

Encapsulation of bFGF and VEGF within hydrogels significantly increases EPC adhesion. The mean number of viable EPCs observed in the hydrogel containing bFGF and VEGF was 378.3 compared with 302.3 in the hydrogels without bFGF and VEGF in hydrogel $(\mathrm{P}<0.05)$. Results suggested that the growth factors significantly increased EPC adhesion.

Encapsulation of bFGF and VEGF within hydrogels significantly increases $M M P$ release. The concentrations of both MMP-2 and MMP-9 in the supernatant were significantly higher for the hydrogels encapsulated with growth factors compared with those without them $(\mathrm{P}<0.05$; Fig. 5).

VEGF and bFGF promotes EPC differentiation. Differentiation determines the success of the implanted EPCs in the transplanted tissue. EPCs were incubated in the hydrogels with or without bFGF and VEGF. Following 7 days of culture, RT-qPCR was used to determine the expression of PECAM1, CD34, KDR, Tek, angiopoietin-2 and pcdh12 genes. Results demonstrated that hydrogels encapsulated with bFGF and VEGF had significantly higher PECAM1, CD34, KDR, Tek, angiopoietin-2 and pcdh12 gene expression compared with hydrogels without bFGF and VEGF $(\mathrm{P}<0.05$; Table II). The significant difference in gene expression demonstrated that VEGF and bFGF promoted the differentiation and maturation of EPCs.

VEGF and bFGF facilitates the neovascularization process. EPCs form new capillaries via vasculogenesis in the center 

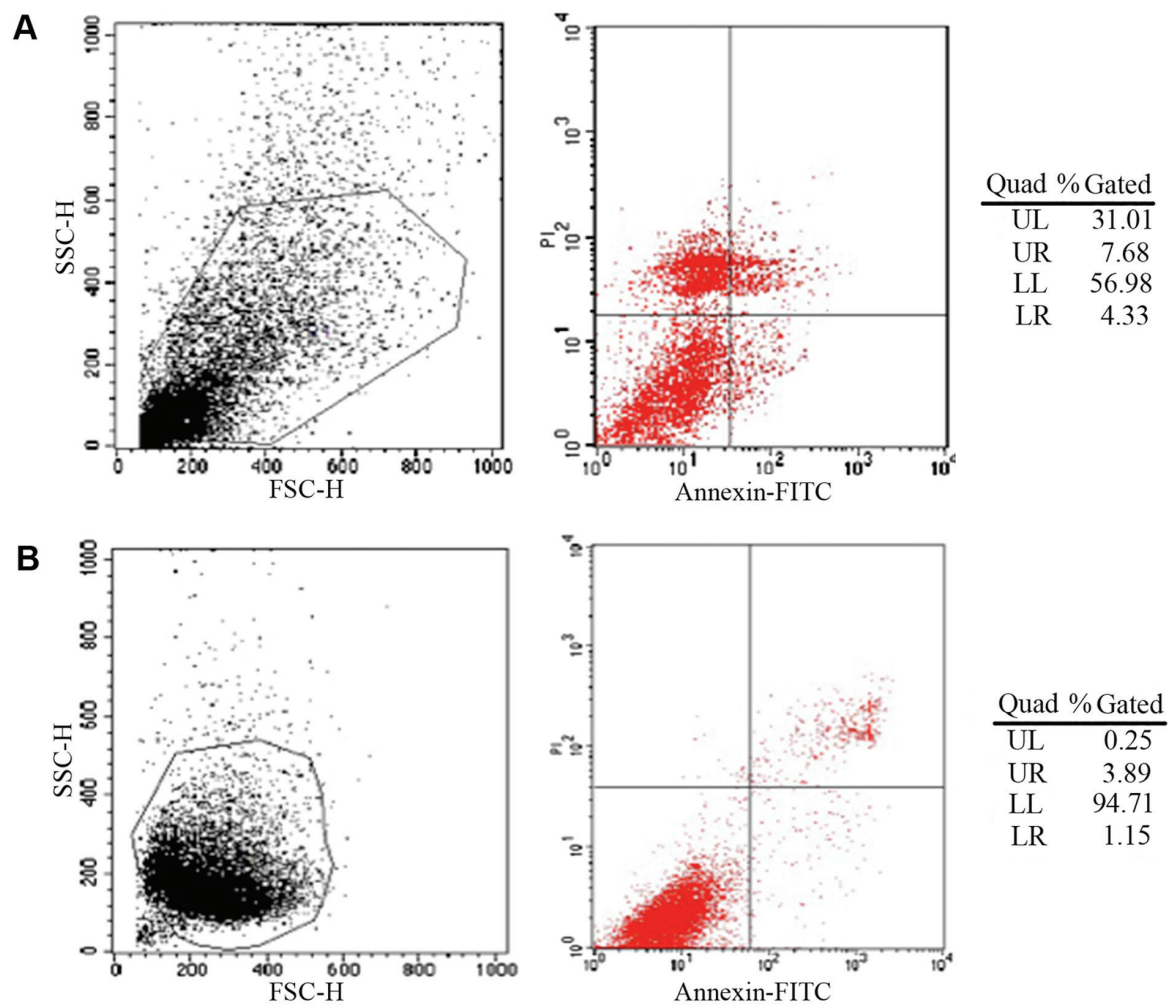

Figure 4. Survival rates of EPCs in PEG hydrogels determined by flow cytometry. (A) PEG hydrogels encapsulated with EPCs, bFGF and VEGF demonstrating $38.69 \%$ surival rate. (B) PEG hydrogels encapsulated with EPCs only demonstrating 4.14\% surival rate. EPC, endothelial progenitor cells; PEG, polyethylene glycol; bFGF, basic fibroblast growth factor; VEGF, vascular endothelial growth factor; UL, upper left; UR, upper right, LL, lower left; LR, lower right; SSC-H, side scatter height; FSC-H, forward scatter height; FITC, fluorescein isothiocyanate; PI, propidium iodide.
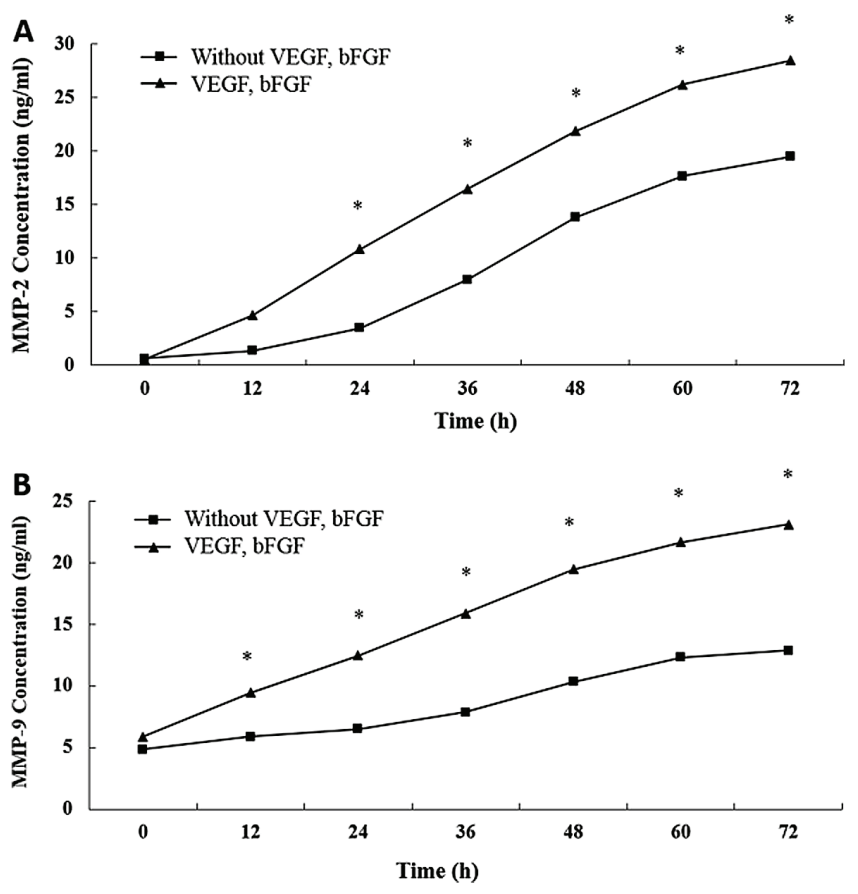

Figure 5. Effect of bFGF and VEGF encapsulation in polyethylene glycol hydrogels on release of MMPs. (A) Release of MMP-2 and of (B) MMP-9 from hydrogels encapsulated with and without bFGF and VEGF. ${ }^{* *} \mathrm{P}<0.05$ vs. control. bFGF, basic fibroblast growth factor; VEGF, vascular endothelial growth factor; MMP, matrix metalloproteinase.

of the infarct (28). By comparison, other primitive cells, such as hematopoietic stem cells, secrete important growth factors such as VEGF, and stimulate healthy small blood vessel generation in the infarcted marginal zone by angiogenesis through 'germination' growth $(29,30)$. Vasculogenesis and angiogenesis work synergistically to establish a new blood supply for the infarction area. In the present study, the CAM assay was used to determine the effect of bFGF and VEGF on angiogenesis induction in vitro. The number of vessels in the hydrogels encapsulated with bFGF and VEGF was significantly higher than the number of vessels in the blank group and hydrogels without bFGF and VEGF encapsulation $(\mathrm{P}<0.05$; Table III). Results indicated that the hydrogel encapsulated with bFGF and VEGF facilitated the neovascularization process, in which both vasculogenesis and angiogenesis are involved.

\section{Discussion}

The present study designed a MMP-sensitive PEG hydrogel modified with RGD then encapsulated bFGF, VEGF and EPCs within the biomaterial to observe and assess its function as a cell transplantation vector and a release system. Hydrogels have good biocompatibility, high water permeability and can be manufactured to produce different microstructures and properties. Therefore, hydrogels can be applied widely in the biomedical field (31). PEG hydrogels display good biocompatibility, histocompatibility and the chemical structure can be adjusted to meet the needs of different biomedical applications $(24,25,32)$. PEG hydrogels also promote the integration of implant and recipient tissue (33). In the current study, inoculated cells were initially suspended to overcome the uneven cell density and weak adhesion. The release of MMPs from cells 
Table II. Endothelial progenitor cell differentiation-associated gene expression.

\begin{tabular}{lcccccc}
\hline Group & PECAM1 & CD34 & KDR & Angio & Tek & Pdch12 \\
\hline With bFGF and VEGF & 1.024 & 1.008 & 1.032 & 0.335 & 1.032 & 1.014 \\
Without bFGF and VEGF & $0.311^{\mathrm{a}}$ & $0.266^{\mathrm{a}}$ & $0.440^{\mathrm{a}}$ & $0.206^{\mathrm{a}}$ & $0.175^{\mathrm{a}}$ & $0.371^{\mathrm{a}}$ \\
\hline
\end{tabular}

${ }^{a} \mathrm{P}<0.05$ vs. With bFGF and VEGF. The two groups were compared using a paired Student's t-test. bFGF, basic fibroblast growth factor; VEGF, vascular endothelial growth factor; PECAM1, platelet/endothelial cell adhesion molecule 1; KDR, kinase insert domain receptor; Tek, TEK receptor tyrosine kinase; angio, angiopoietin-2; Pdch12, protocadherin.

Table III. Chick chorioallantoic membrane angiogenesis assay.

\begin{tabular}{lcccr}
\hline Group & Large blood vessels & Medium blood vessels & Small blood vessels & Total \\
\hline Blank control & 1 & 5 & 3 & $9^{\mathrm{a}}$ \\
PEG hydrogel without bFGF and VEGF & 2 & 7 & 16 & $25^{\mathrm{a}}$ \\
PEG hydrogel with bFGF and VEGF & 4 & 10 & 26 & 36 \\
\hline
\end{tabular}

${ }^{\mathrm{a} P}<0.05$ vs. PEG hydrogel with bFGF and VEGF. PEG, polyethylene glycol; bFGF, basic fibroblast growth factor; VEGF, vascular endothelial growth factor.

gradually degraded the hydrogel therefore cell proliferation and hydrogel degradation had a combined effect. The modified PEG hydrogel system serves an important role in assisting and protecting against the above process. Results indicated that the initial hydrogel structure was maintained for 7 days, which makes it appropriate for a role as a sustained release carrier.

RGD is one of the smallest amino acid sequence recognized by many adherent proteins (8). This sequence binds specifically to cell surface integrin receptors, thereby mediating cell adhesion, migration and growth (34-37). Directly immobilizing RGD to the surface of a material can promote the adhesion of the receptor-mediated cells. In the present study, EPCs were isolated from rat bone marrow cells with this cell source excluding the possibility of contamination from vascular ECs. Semi-quantitative PCR for EPC related markers confirmed successful EPC isolation. Several other robust tools for confirmation of EPC isolation include western blot analysis, phase contrast microscopy and morphological assessment. The average number of EPCs observed on the surface of the RGD-modified hydrogel group was significantly higher compared with the non-RGD modified group. In addition, EPCs were determined to rapidly adhere to the hydrogel with the cells and material forming contacting points. EPCs dispersed radially in the modified hydrogels. The RGD-modified hydrogel significantly increased the adhesion and proliferation of EPCs, which suggested that the presence of RGD significantly promoted biocompatibility.

Many studies have identified VEGF and bFGF as the most important growth factors for therapeutic angiogenesis $(12,13)$. However, maintaining EPC activity and integration with host blood vessels, and ensuring sustained release of vascular growth factor in the wound long-term have not yet been solved in angiogenesis research. Therefore, in the present study, hydrogels were encapsulated with bFGF, VEGF and EPCs to determine the sustained release effect of bFGF and VEGF, and also to observe the culture of EPCs. Following addition of MMP-2 and MMP-9 at $72 \mathrm{~h}$, the release \% of bFGF and VEGF increased steadily and reached $>95 \%$. These results suggested that the MMP-sensitive PEG hydrogels released bFGF and VEGF in a stable manner. The results also imply that VEGF and bFGF have a synergistic role in the formation of neovascularization. In addition, following 7 days of culture, 38.69\% of EPCs survived, suggesting that the modified hydrogels supported EPC proliferation, reduced apoptosis and improved the survival rate.

VEGF and bFGF participate in all aspects of angiogenesis and promote the degradation of capillary basement membrane, facilitate EC proliferation and migration, and the formation of tubular structures (38). This process eventually leads to capillary angiogenesis and microcirculation improvement (39). The effect of bFGF and VEGF on the proliferation, differentiation, maturation and vascularization of EPCs in hydrogels has yet to be fully elucidated therefore the present study investigated encapsulation of EPC and growth factors within a PEG hydrogel. RGD-modified hydrogels encapsulated with VEGF and bFGF displayed $\sim 40 \%$ EPCs attachment at 7 days compared with $\sim 4 \%$ EPC attachment for RGD hydrogels without VEGF and bFGF. These results suggested that VEGF and bFGF enhanced the adhesion of EPCs, and facilitated the proliferation of EPCs. Gene expression analysis determined that EPC-related gene expression was higher in hydrogels encapsulated with bFGF and VEGF compared with the group without bFGF and VEGF. These results suggested that VEGF and bFGF promoted the differentiation and maturation of EPCs.

There are several cell types suitable for angiogenesis studies, including HUVECs and EPCs. In the present study, EPCs were chosen due to their important roles in both angiogenesis and vasculogenesis, as well as their ability to migrate into the blood system stream and ability to differentiate into a variety of mature vascular EC types. The concentration of VEGF in vitro is positively correlated with the proliferation 
rate of EPCs (40-43). bFGF can facilitate EC proliferation and migration. When bFGF functions together with VEGF, this synergistic pair promotes the splitting of ECs into capillary-like structures inducing angiogenesis in vivo $(44,45)$. For the present CAM study, new blood vessels grew markedly. The number of blood vessels in the bFGF and VEGF group was significantly higher than the control group. These results suggested that the hydrogels containing bFGF and VEGF induced CAM angiogenesis. Sustained release of bFGF and VEGF is capable of stimulating EC proliferation and budding, but also capable of promoting transplantation of EPCs hyperplasia and vascularization. Transplanted EPCs are able to overcome the limitations of EC aging and dysfunction, indicating that the two components in the hydrogel have a strong synergistic association.

In conclusion, the RGD-modified hydrogel displayed good mechanical properties suitable for a role as a sustained release carrier. The presence of RGD in the hydrogel significantly promoted its biocompatibility as EPC proliferation was supported, apoptosis was reduced and EPC survival rate was improved. In addition, hydrogels encapsulated with VEGF and bFGF enhanced the adhesion of ECs, promoted the production of extracellular matrices and facilitated EPC proliferation. The differentiation and maturation of EPCs were also facilitated in the RGD-modified hydrogels. Furthermore, the hydrogels encapsulated with bFGF and VEGF induced angiogenesis with the two growth factors and EPCs displaying a strong synergy. The present results may provide a potential treatment for soft tissue defects such as bone exposure, chronic skin ulcers, bedsores, limb necrosis, osteonecrosis and other ischemic diseases.

\section{Acknowledgements}

Not applicable.

\section{Funding}

The present study was supported by the National Key R\&D Program of China (grant no. 2017YFC1103800) and Natural Science Fund of Hubei (grant no. 2016CFB657).

\section{Availability of data and materials}

The datasets used and/or analyzed during the current study are available from the corresponding author on reasonable request.

\section{Authors' contribution}

LO, YD and DD conceived of the current study. LO and YD designed the current study. LO, YD, ZS, GL and SY obtained the relevant data. CY, GL and DD drafted the manuscript. CY revised the manuscript critically for important intellectual content. All authors analyzed and interpreted the data, and also read and approved the final manuscript.

\section{Ethics approval and consent to participate}

Protocols involving the use of the animals were approved by the Ethics Committee of Tongji Medical College, Huazhong University of Science and Technology (2016 IACUC Number: S782). Animal experiments were performed in accordance with the Guide for the Care and Use of Laboratory Animals.

\section{Patient consent for publication}

Not applicable.

\section{Competing interests}

The authors declare that they have no competing interests.

\section{References}

1. Winkler H: Treatment of chronic orthopaedic infection. EFORT Open Rev 2: 110-116, 2017.

2. Fadini GP, Losordo D and Dimmeler S: Critical reevaluation of endothelial progenitor cell phenotypes for therapeutic and diagnostic use. Circ Res 110: 624-637, 2012.

3. Asahara T, Murohara T, Sullivan A, Silver M, van der Zee R, Li T, Witzenbichler B, Schatteman G and Isner JM: Isolation of putative progenitor endothelial cells for angiogenesis. Science 275: 964-967, 1997

4. Janic B and Arbab AS: The role and therapeutic potential of endothelial progenitor cells in tumor neovascularization. ScientificWorldJouralo 10: 1088-1099, 2010.

5. Khoo CP, Pozzilli $P$ and Alison MR: Endothelial progenitor cells and their potential therapeutic applications. Regen Med 3: 863-876, 2008

6. Sun Q, Silva EA, Wang A, Fritton JC, Mooney DJ, Schaffler MB, Grossman PM and Rajagopalan S: Sustained release of multiple growth factors from injectable polymeric system as a novel therapeutic approach towards angiogenesis. Pharm Res 27: 264-271, 2010.

7. Marfella R, Luongo C, Coppola A, Luongo M, Capodanno P, Ruggiero R, Mascolo L, Ambrosino I, Sardu C, Boccardi V, et al: Use of a non-specific immunomodulation therapy as a therapeutic vasculogenesis strategy in no-option critical limb ischemia patients. Atherosclerosis 208: 473-479, 2010.

8. Benton JA, Fairbanks BD and Anseth KS: Characterization of valvular interstitial cell function in three dimensional matrix metalloproteinase degradable PEG hydrogels. Biomaterials 30 : 6593-6603, 2009.

9. Brandl F, Kastner F, Gschwind RM, Blunk T, Tessmar J and Gopferich A: Hydrogel-based drug delivery systems: Comparison of drug diffusivity and release kinetics. J Control Release 142: 221-228, 2010.

10. Hanjaya-Putra D, Yee J, Ceci D, Truitt R, Yee D and Gerecht S: Vascular endothelial growth factor and substrate mechanics regulate in vitro tubulogenesis of endothelial progenitor cells. J Cell Mol Med 14: 2436-2447, 2010.

11. Wang QR, Wang BH, Huang YH, Dai G, Li WM and Yan Q: Purification and growth of endothelial progenitor cells from murine bone marrow mononuclear cells. J Cell Biochem 103: 21-29, 2008.

12. Kraehenbuehl TP, Ferreira LS, Zammaretti P, Hubbell JA and Langer R: Cell-responsive hydrogel for encapsulation of vascular cells. Biomaterials 30: 4318-4324, 2009.

13. Mieno S, Boodhwani M, Robich MP, Clements RT, Sodha NR and Sellke FW: Effects of diabetes mellitus on VEGF-induced proliferation response in bone marrow derived endothelial progenitor cells. J Card Surg 25: 618-625, 2010

14. Sufen G, Xianghong Y, Yongxia C and Qian P: bFGF and PDGF-BB have a synergistic effect on the proliferation, migration and VEGF release of endothelial progenitor cells. Cell Biol Int 35: 545-551, 2011.

15. Seliktar D, Zisch AH, Lutolf MP, Wrana JL and Hubbell JA: MMP-2 sensitive, VEGF-bearing bioactive hydrogels for promotion of vascular healing. J Biomed Mater Res A 68A: 704-716, 2004.

16. Yeo Y, Geng W, Ito T, Kohane DS, Burdick JA and Radisic M: Photocrosslinkable hydrogel for myocyte cell culture and injection. J Biomed Mater Res B Appl Biomater 81: 312-322, 2007.

17. Kraehenbuehl TP, Zammaretti P, Van der Vlies AJ, Schoenmakers RG, Lutolf MP, Jaconi ME and Hubbell JA: Three-dimensional extracellular matrix-directed cardioprogenitor differentiation: Systematic modulation of a synthetic cell-responsive PEG-hydrogel. Biomaterials 29: 2757-2766, 2008. 
18. Wang X, Zhao Z, Zhang H, Hou J, Feng W, Zhang M, Guo J, Xia J, Ge Q, Chen X and Wu X: Simultaneous isolation of mesenchymal stem cells and endothelial progenitor cells derived from murine bone marrow. Exp Ther Med 16: 5171-5177, 2018

19. Huizer K, Mustafa DAM, Spelt JC, Kros JM and Sacchetti A: Improving the characterization of endothelial progenitor cell subsets by an optimized FACS protocol. PLos One 12: e0184895, 2017.

20. Camci-Unal G, Nichol JW, Bae H, Tekin H, Bischoff J and Khademhosseini A: Hydrogel surfaces to promote attachment and spreading of endothelial progenitor cells. J Tissue Eng Regen Med 7: 337-347, 2013.

21. Livak KJ and Schmittgen TD: Analysis of relative gene expression data using real-time quantitative PCR and the 2(-Delta Delta C(T)) method. Methods 25: 402-408, 2001

22. Valdes TI, Kreutzer D and Moussy F: The chick chorioallantoic membrane as a novel in vivo model for the testing of biomaterials. J Biomed Mater Res 62: 273-282, 2002.

23. Deryugina EI and Quigley JP: Chapter 2: Chick embryo chorioallantoic membrane models to quantify angiogenesis induced by inflammatory and tumor cells or purified effector molecules. Methods Enzymol 444: 21-41, 2008.

24. Hill-West JL, Chowdhury SM, Slepian MJ and Hubbell JA: Inhibition of thrombosis and intimal thickening by in situ photopolymerization of thin hydrogel barriers. Proc Natl Acad Sci USA 91: 5967-5971, 1994.

25. West JL and Hubbell JA: Separation of the arterial wall from blood contact using hydrogel barriers reduces intimal thickening after balloon injury in the rat: The roles of medial and luminal factors in arterial healing. Proc Natl Acad Sci USA 93: 13188-13193, 1996.

26. Reinlib L and Field L: Cell transplantation as future therapy for cardiovascular disease?: A workshop of the National Heart, Lung, and Blood Institute. Circulation 101: E182-E187, 2000.

27. Kawamoto A and Losordo DW: Endothelial progenitor cells for cardiovascular regeneration. Trends Cardiovasc Med 18: 33-37, 2008.

28. Shintani S, Murohara T, Ikeda H, Ueno T, Honma T, Katoh A, Sasaki K, Shimada T, Oike Y and Imaizumi T: Mobilization of endothelial progenitor cells in patients with acute myocardial infarction. Circulation 103: 2776-2779, 2001.

29. Richardson TP, Peters MC, Ennett AB and Mooney DJ: Polymeric system for dual growth factor delivery. Nat Biotechnol 19 1029-1034, 2001

30. Arras M, Mollnau H, Strasser R, Wenz R, Ito WD, Schaper J and Schaper W: The delivery of angiogenic factors to the heart by microsphere therapy. Nat Biotechnol 16: 159-162, 1998.

31. Heldman AW, Cheng L, Jenkins GM, Heller PF, Kim DW, Ware M Jr, Nater C, Hruban RH, Rezai B, Abella BS, et al: Paclitaxel stent coating inhibits neointimal hyperplasia at 4 weeks in a porcine model of coronary restenosis. Circulation 103: 2289-2295, 2001.

32. Slepian MJ: Polymeric endoluminal paving: A family of evolving methods for extending endoluminal therapeutics beyond stenting. Cardiol Clin 12: 715-737, 1994.
33. Zhang X, Xu B, Puperi DS, Wu Y, West JL and Grande-Allen KJ: Application of hydrogels in heart valve tissue engineering. J Long Term Eff Med Implants 25: 105-134, 2015.

34. Eichler W, Friedrichs U, Thies A, Tratz C and Wiedemann P. Modulation of matrix metalloproteinase and TIMP-1 expression by cytokines in human RPE cells. Invest Ophthalmol Vis Sci 43: 2767-2773, 2002

35. $\mathrm{Ma} \mathrm{C}$ and Chegini N: Regulation of matrix metalloproteinases (MMPs) and their tissue inhibitors in human myometrial smooth muscle cells by TGF-beta1. Mol Hum Reprod 5: 950-954, 1999.

36. Overall CM, Wrana JL and Sodek J: Transcriptional and post-transcriptional regulation of 72-kDa gelatinase/type IV collagenase by transforming growth factor-beta 1 in human fibroblasts. Comparisons with collagenase and tissue inhibitor of matrix metalloproteinase gene expression. J Biol Chem 266: 14064-14071, 1991

37. Wick W, Platten M and Weller M: Glioma cell invasion: Regulation of metalloproteinase activity by TGF-beta. J Neurooncol 53: 177-185, 2001.

38. Guan J, Sacks MS, Beckman EJ and Wagner WR: Synthesis, characterization, and cytocompatibility of elastomeric, biodegradable poly(ester-urethane)ureas based on poly(caprolactone) and putrescine. J Biomed Mater Res 61: 493-503, 2002.

39. Ferrara $\mathrm{N}$ and Alitalo K: Clinical applications of angiogenic growth factors and their inhibitors. Nat Med 5: 1359-1364, 1999.

40. Elbert DL and Hubbell JA: Conjugate addition reactions combined with free-radical cross-linking for the design of materials for tissue engineering. Biomacromolecules 2: 430-441, 2001.

41. Henry TD, Rocha-Singh K, Isner JM, Kereiakes DJ, Giordano FJ, Simons M, Losordo DW, Hendel RC, Bonow RO, Eppler SM, et al: Intracoronary administration of recombinant human vascular endothelial growth factor to patients with coronary artery disease. Am Heart J 142: 872-880, 2001.

42. Lee KY, Peters MC, Anderson KW and Mooney DJ: Controlled growth factor release from synthetic extracellular matrices. Nature 408: 998-1000, 2000.

43. Mann BK, Gobin AS, Tsai AT, Schmedlen RH and West JL: Smooth muscle cell growth in photopolymerized hydrogels with cell adhesive and proteolytically degradable domains: Synthetic ECM analogs for tissue engineering. Biomaterials 22: 3045-3051, 2001.

44. Lutolf MP and Hubbell JA: Synthesis and physicochemical characterization of end-linked poly(ethylene glycol)-co-peptide hydrogels formed by michael-type addition. Biomacromolecules 4 : 713-722, 2003.

45. West JL and Hubbell JA: Polymeric biomaterials with degradation sites for proteases involved in cell migration. Macromolecules 32: 241-244, 1999.

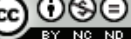

This work is licensed under a Creative Commons Attribution-NonCommercial-NoDerivatives 4.0 International (CC BY-NC-ND 4.0) License. 